\title{
Dynamic Propagation Problems of Mode III Semi-Infinite Crack
}

\author{
${ }^{1}$ Lu Nian-Chun, ${ }^{2}$ Li Xin-Gang, ${ }^{3}$ Cheng Yun-Hong and ${ }^{4}$ Cheng Jin \\ ${ }^{1}$ Department of Material Science and Engineering, Shenyang Ligong University, Shenyang 110159, P R China \\ ${ }^{2}$ Department of Civil Engineering, Harbin Engineering University, Harbin 150001, P R China \\ ${ }^{3}$ Department of Civil Engineering, Northeastern University, Shenyang 110006, P R China \\ ${ }^{4}$ Department of Astronautics and Mechanics, Harbin Institute of Technology, Harbin 150001, P R China
}

Received 2012-12-04, Revised 2013-04-11; Accepted 2013-07-08

\begin{abstract}
By means of the theory of complex functions, dynamic propagation problems of mode III semi- infinite crack were researched. The problems studied can be facilely transformed into riemann-hilbert problems and the universal expression of analytical solutions of stress, displacement and dynamic stress intensity factor under the conditions of moving increasing loads $\mathrm{Px} / \mathrm{t}$ and $\mathrm{Pt}^{2} / \mathrm{x}$, respectively, are very facilely obtained using the measures of self-similar functions. In view of corresponding material properties, the mutative rule of dynamic stress intensity factor was illuminated very well.
\end{abstract}

Keywords: Complex Functions, Dynamic Propagation Problems, Mode III Semi-Infinite Crack, SelfSimilar Functions Analytical Solutions

\section{INTRODUCTION}

For an orthotropic anisotropic body, let the Cartesian co-ordinates be coincident with the axes of elastic symmetry. The considered problems are confined in the anti-plane. The anti-plane equation of motion for an orthotropic anisotropic body is given by (Eringer, 1975; Sih, 1977a; Erdogan, 1968; Cherepanov, 1979; Kostrov, 1964; Freund, 1998): Statics problems of anti-plane shear loading were researched very much (Eringer, 1975; Sih, 1977a; Erdogan, 1968) in the past of several decades. Dynamic problem is an important research field in modern fracture mechanics. Due to the interaction of the stress waves under complex dynamic loading cases, the theory of dynamic fracture mechanics is still innovated and developed (Cherepanov, 1979; Sih, 1968; Freund, 1998). However, a lot of engineering structures are under the conditions of dynamic loadings and the static theory can't effectually III resolve a series of dynamic queries, so it is indispensable to study the fracture dynamics problems (Sih, 1968; Kostrov, 1964; Freund, 1998; Sih and MacDonald, 1974; Srolovitz and Source, 1997). A lot of researches on mode III crack dynamic propagation problems were performed cautiously (Freund, 1998; Sih and MacDonald, 1974; Srolovitz and Source, 1997; Gao, 1996; Andrew, 1976; Rosakis et al., 1999; Tang and Sih, 2004). In view of the complexity, cockamamie and difficulty in mathematical operations, fracture dynamics problems are not investigated enough thoroughly (Sih, 1968; Kostrov, 1964; Freund, 1998; Sih and MacDonald, 1974; Srolovitz and Source, 1997; Broberg, 1960; RubinGonzalea and Mason, 2000) and numerical solutions acquired (Sih and MacDonald, 1974; Sih, 1973; 1991; Fedelinski et al., 1997; Wang et al., 1992; Knauss, 1987; Wang, 1992; Ranjith and Narasimhan, 1996) were much more than analytical solutions (Nian-Chun et al., 2004; 2005; 2006). On account of those situations mentioned above, therefore it is necessary to study dynamic propagation problem concerning mode III semi-infinite crack. Universal expressions of analytical solutions are given by means of the theory of complex functions. The problems studied can be easily transformed into a RiemannHilbert problem utilizing method (Muskhelishvili, $1953 ;$ 1977). The study in this study is how to Corresponding Author: Lu Nian-Chun, Department of Material Science and Engineering, Shenyang Ligong University,
Shenyang 110159, P R China 
expediently obtain analytical solutions using the selfsimilar functions under the action of moving increasing loads.

\subsection{Correlative Equations of the Anti-Plane Problem Concerning Elastodynamics for an Orthotropic Anisotropic Body}

For an orthotropic anisotropic body, let the Cartesian co-ordinates be coincident with the axes of elastic symmetry. The considered problems are confined in the anti-plane. The anti-plane equation of motion for an orthotropic anisotropic body is given by (Eringer, 1975; Sih, 1977a; Erdogan, 1968; Cherepanov, 1979; Kostrov, 1964; Freund, 1998):

$\mathrm{C}_{55} \frac{\partial^{2} \mathrm{w}}{\partial \mathrm{x}^{2}}+\mathrm{C}_{44} \frac{\partial^{2} \mathrm{w}}{\partial \mathrm{y}^{2}}=\rho \frac{\partial^{2} \mathrm{w}}{\partial \mathrm{t}^{2}}$

where, $\mathrm{C}_{44}, \mathrm{C}_{55}$ are the elastic constants, $\rho$ is the mass density and $\mathrm{W}$ is the displacement component along $\mathrm{Z}$ (Cherepanov, 1979; Sih, 1968; Sih and MacDonald, 1964; Freund, 1998; Nian-Chun et al., 2004; 2005; 2006; Atkinson, 1975; 1965).

Using Atkinson transform (Nian-Chun et al., 2004; 2005; 2006; Atkinson, 1975; 1965), it is found that Equation 2:

$\xi=x-\eta t+T y$

where, $\eta$ is to be understood as a complex variable and $\mathrm{T}$ is a function of $\eta$.

A solution of equation of motion can be constructed as:

$$
\mathrm{w}=\operatorname{Re}\left[\int_{-\infty}^{\infty} \varphi(\xi) \mathrm{d \eta}\right]
$$

where, the integral is the real part of $\eta$-axis.

Now putting Equation 3 into 1, the following representation must exist:

$$
\mathrm{C}_{55}+\mathrm{C}_{44} \mathrm{~T}^{2}-\rho \eta^{2}=0
$$

Equation 1 will become identical equation, hence $\phi(\xi)$ is a discretionary function to be determined from the boundary conditions.

Postulating that Equation 4 has two complex roots, then we only take the imaginary part with positive sign, i.e., positive square root. When
$T(\eta)=-i \sqrt{\left(C_{55}-\rho \eta^{2}\right) / C_{44}}$ exists, the measure of solution is the same as that of $\mathrm{T}(\eta)=\mathrm{i} \sqrt{\left(\mathrm{C}_{55}-\rho \eta^{2}\right) / \mathrm{C}_{44}}$, in order to represent expediently, therefore the positive root is taken. Here exists (Cherepanov, 1979; Nian-Chun et al., 2004; 2005; 2006; Atkinson, 1975):

$\mathrm{T}(\eta)=\mathrm{i} \sqrt{\left(\mathrm{C}_{55}-\rho \eta^{2}\right) / \mathrm{C}_{44}}$

At $\mathrm{y}=0$, one attains the universal conclusions (Cherepanov, 1979; Nian-Chun et al., 2004; 2005; 2006; Atkinson, 1975; 1965):

When functions $\mathrm{L}_{\mathrm{w}}$ is homogeneous, there results:

$\mathrm{w}^{0}=\mathrm{Lw}, \tau_{\mathrm{x} 2}^{0}=\mathrm{L} \tau_{\mathrm{xz}}, \tau_{\mathrm{y} 2}^{0}=\mathrm{L} \tau_{\mathrm{yz}}$

When $L \tau_{\mathrm{x} 2}$ and $L \tau_{\mathrm{y} 2}$ are homogeneous, there results Equation 7:

$\mathrm{w}^{0}=\frac{\partial}{\partial \mathrm{t}} \mathrm{Lw}, \tau_{\mathrm{x} 2}^{0}, \frac{\partial}{\partial \mathrm{t}} \mathbf{L} \tau_{\mathrm{xz}}, \tau_{\mathrm{y} 2}^{0} \frac{\partial}{\partial \mathrm{t}} \mathbf{L} \tau_{\mathrm{y} 2}$

where, $\mathrm{L}=\frac{\partial^{\mathrm{m}+\mathrm{n}}}{\partial \mathrm{x}^{\mathrm{m}} \partial \mathrm{t}^{\mathrm{n}}}$, inverse: $\mathrm{L}^{-}=\frac{\partial^{-\mathrm{m}-\mathrm{n}}}{\partial \mathrm{x}^{-\mathrm{m}} \partial \mathrm{t}^{-\mathrm{n}}}$. Here $+\mathrm{m}+\mathrm{n}$, $-m-n$ and 0 represent the $(m+n)$ th order derivative, the $(m+n)$ th order integral and function's self, respectively. The coefficients $\mathrm{m}, \mathrm{n}$ will be called the indices of self-similarity (Cherepanov, 1979; Nian-Chun et al., 2004; 2005; 2006; Atkinson, 1975; 1965).

Utilizing relevant Representations of the anti-plane problem concerning elastodynamics equations of motion for an orthotropic anisotropic body, the general expressions can be rewritten in the following modality (Cherepanov, 1979; Nian-Chun et al., 2004; 2005; 2006; Atkinson, 1975; 1965):

$\tau_{\mathrm{yz}}^{0}=\frac{1}{\mathrm{t}} \operatorname{Re}[\mathrm{f}(\tau) \mathrm{T}(\tau)], \tau_{\mathrm{xz}}=\frac{\mathrm{C}_{55}}{\mathrm{C}_{44} \mathrm{t}} \operatorname{Ref}(\tau), \frac{\partial \mathrm{w}^{0}}{\partial \tau}=\frac{1}{\mathrm{C}_{44}} \operatorname{Ref}(\tau)$

where, $\tau=\mathrm{x} / \mathrm{t}, \mathrm{f}(\tau)$ is self-similar functions. The values of $\mathrm{T}(\tau)$ can be ascertained from Equation 5 . Indicated that $\mathrm{T}(\tau)$ in the subsonic speeds is purely imaginary for the considered values. Thus, elastodynamics problems for an orthotropic anisotropic body can be translated into the sole unknown function problems of $\mathrm{f}(\tau)$ satisfying the boundary-value conditions. 


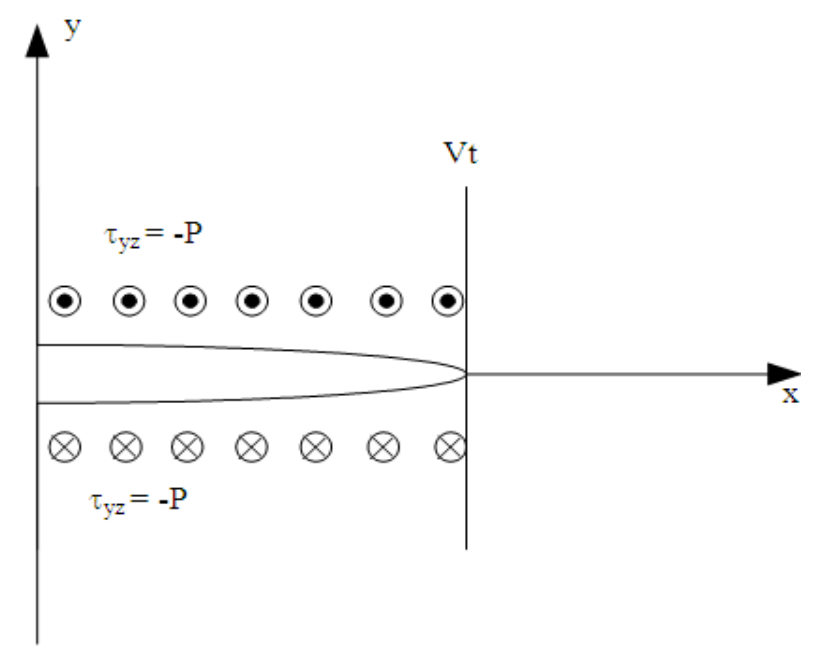

Fig. 1. Schematic of dynamic semi-infinite crack propagation of orthotropic solids under anti-plane shear loading

In the general case this is Riemann-Hilbert problem in the theory of complex functions, for the simplest case, which is the Keldysh-Sedov or dirchlet problem (Muskhelishvili, 1953; 1977).

\subsection{Indication of Dynamic Semi-Infinite Crack Propagation of Orthotropic Solids under Anti-Plane Shear Loading}

Assume at the initial moment $\mathrm{t}=0$, a crack according to my presumption begins to nucleate from an infinitesimally small micro-crack under the action of shear loads $\mathrm{P}$ located at the point of infinity, running with constant velocity $\mathrm{V}$ (for the subsonic speeds) in the positive direction of $\mathrm{x}$-axis. The schematic of dynamic semi-infinite crack propagation of orthotropic solids under anti-plane shear loading is depicted in Fig. 1. The configuration is symmetry both in geometry and mechanics with respect to $\mathrm{x}$-axis, but it is asymmetry with respect to y-axis in virtue of crack semi-infinite extension. The crack locates in the domain of $\mathrm{y}=0,0<\mathrm{x}<\mathrm{Vt}$ moreover closed force $\mathrm{P}$ acts on this segment, whose magnitude is unknown, unascertained. The force expresses shear stress $\tau$ lying in the rear segment of the crack tip. When the crack moves at high speed, its dimension must relate to variables $\mathrm{x}$ and $\mathrm{t}$, then the crack edges subjected to loads also have relation to variables $\mathrm{x}$ and $\mathrm{t}$.

\subsection{Rudimental Solution of Dynamic Extension for Semi-Infinite Crack Problem}

Postulated at the initial moment $\mathrm{t}=0$, a microcrack suddenly occurs at an orthotropic anisotropic body, the Cartesian co-ordinate axes is introduced which accords with the axes of elastic symmetry of the body and the motion of the crack is restricted in the $x-\square y$ - $\square$ plane at constant velocity $\mathrm{V}$ along the positive direction of $x$-axis. The relative following boundary condition problems will be depicted as Equation 9:

$\tau_{\mathrm{yz}}(\mathrm{x}, 0, \mathrm{t})=\mathrm{g}_{1}(\mathrm{x}, \mathrm{t}), 0<\mathrm{x}<\mathrm{Vt}, \mathrm{w}(\mathrm{x}, 0, \mathrm{t})=0, \mathrm{x}>\mathrm{Vt}$

Introducing the variable $\tau=\mathrm{x} / \mathrm{t}$. In terms of Equation 8 and $\mathrm{t} \delta(\mathrm{x})=\delta(\mathrm{x} / \mathrm{t})$ in the theory of generalized functions (Hoskins, 1979; Gahov, 1966), the mentioned-above boundary conditions can be readily translated into the undermentioned boundary value queries Equation 10:

$\operatorname{Re}[\mathrm{f}(\tau) \mathrm{T}(\tau)]=\mathrm{g}_{2}[\tau, \delta(\tau)], 0<\tau<\operatorname{VRef}(\tau)=0 \tau>\mathrm{V}$

In terms of Equation 8 and the previous conditions, the sole unknown function $\mathrm{f}(\tau)$ must have the following Equation 11:

$\mathrm{f}(\tau)=\mathrm{g}_{3}[(\tau, \xi(\tau)]$

Functions of $g_{1}, g_{2}$ and $g_{3}$ represent different functions in concrete mathematical operations in order to avoid confusion with alphabet "f" in formulas (9)-(11), so I use "g" instead " $\mathrm{f}$ ".

Then the problem considered can be come down to Keldysh-Sedov problem as Equation 12:

$\operatorname{Rem}(\tau)=0, \tau>\mathrm{V}, \operatorname{Imm}(\tau)=0,0<\tau<\mathrm{V}$

Considering dissymmetry and the conditions of the infinite point of the plane corresponding to the origin of coordinates of the physical plane as well as singularities of the stress at the crack tip (Gahov, 1966; Sih, 1977b; Kanwal and Sharma, 1976), the rudimental solution of the above problems can be obtained as follows Equation 13:

$\xi(\tau)=\mathrm{T}[(\mathrm{V}-\tau), \tau]$

Afterwards applying Equation 8 and 5, the stress, the displacement and the stress intensity factor under the conditions of semi-infinite crack dynamic expansion problems will be easily derived.

\subsection{The Solutions of Concrete Problems}


In order to resolve effectually fracture dynamics problems on an orthotropic anisotropic body, analytic solutions will be obtained under the action of variable loads for mode III semi-infinite crack. In terms of the principle of generalized functions, the unlike boundary condition problems will be facilely transformed into Keldysh-Sedov mixed boundary value problem by means of self-similar functions and the corresponding solutions will be gained.

Presumed at the initial moment $\mathrm{t}=0$, a microcrack is abruptly initiated and begins to run at constant velocity $\mathrm{V}$ along the positive direction of $\mathrm{x}$ axis. The crack surfaces are subjected to normal point force $\mathrm{Px} / \mathrm{t}$, moving at a constant velocity $\beta<\mathrm{V}$ along the positive direction of $\mathrm{x}$-axis. On the half-plane at $\mathrm{y}$ $=0$, the boundary conditions will be shown as follows Equation 14:

$$
\begin{aligned}
& \tau_{\mathrm{yz}}(\mathrm{x}, 0, \mathrm{t})=-\mathrm{Px} / \mathrm{t} . \delta(\mathrm{x}-\beta \mathrm{t}), 0<\mathrm{x}<\mathrm{Vt} \\
& \mathrm{w}(\mathrm{x}, 0, \mathrm{t})=0, \quad \mathrm{x}>\mathrm{Vt}
\end{aligned}
$$

Evidently the displacement will be homogeneous functions, in which $L=1$. Using variable $\tau=x / t$ and the theory of generalized functions (Hoskins, 1979; Gahov, 1966) as well as Equation 8 and 6, the first of Equation 14 can be rewritten as:

$$
\operatorname{Re}[\mathrm{T}(\tau) \mathrm{f}(\tau)]=-\mathrm{Px} / \mathrm{t} . \delta(\mathrm{x}-\beta \mathrm{t})=-\mathrm{P} \tau . \delta(\tau-\beta), \quad 0<\tau<\mathrm{V}
$$

Deducting from the above formulae, the unique solution of $f(\tau)$ must have this format:

$$
f(\tau)=\tau \cdot \xi(\tau) /(\tau-\beta)
$$

In the formula $\xi(\tau)$ has no singularity in the domain of $0<\tau<\mathrm{V}$, while $\mathrm{T}(\tau)$ is purely imaginary for the subsonic speeds; hence $\xi(\tau)$ must be purely real in the region of $0<\tau<\mathrm{V}$. Thus, Equation 16 becomes Equation 17:

$\operatorname{Re} \xi(\tau)=0, \tau>\mathrm{V}, \operatorname{Im} \xi(\tau)=0,0<\tau<\mathrm{V}$

In terms of asymmetry and the conditions of the infinite point of the plane corresponding to the origin of coordinates of the physical plane as well as singularities of the crack tip (Gahov, 1966; Sih, 1977b; Kanwal and Sharma, 1976) the unique solution of the Keldysh-Sedov problem (17) can be attained:

$$
\xi(\tau)=\frac{\mathrm{A}}{\sqrt{(\mathrm{V}-\tau) \tau}}
$$

where, $\mathrm{A}$ is an unknown constant.

Substituting Equation 18 into 16, one can gain:

$$
f(\tau)=\frac{A \tau}{(\tau-\beta) \sqrt{(V-\tau) \tau}}
$$

Putting Equation 19, 5 into Equation 15, at $\tau \rightarrow \beta$, constant A will be ascertained as follows Equation 20:

$$
A=-\frac{P \sqrt{(V-\beta) \beta}}{\pi \sqrt{\left(C_{55}-\rho \beta^{2}\right) / C_{44}}}
$$

Afterwards Equation 19 and 5 may be replaced into Equation 6 and 8 , at the surface $y=0$, the stress $\tau_{\mathrm{yz}}$, the displacement $\mathrm{W}$ and stress intensity factor $\mathrm{K}_{3}(\mathrm{t})$ are obtained, respectively Equation 21 and 22:

$$
\begin{aligned}
& \tau_{\mathrm{yz}}=\tau_{\mathrm{yz}}^{0}=\frac{1}{\mathrm{t}} \operatorname{Re}[\mathrm{f}(\tau) \mathrm{T}(\tau)]=\frac{1}{\mathrm{t}} \cdot \operatorname{Re} \frac{\mathrm{A} \cdot \tau \cdot \mathrm{T}(\tau)}{(\tau-\beta) \sqrt{(\tau-\mathrm{V}) \tau}} \\
& =\frac{\mathrm{A} \tau \sqrt{\left(\mathrm{C}_{55}-\rho \tau^{2}\right) / \mathrm{C}_{44}}}{(\tau-\beta) \sqrt{(\mathrm{x}-\mathrm{Vt}) \mathrm{x}}}=\frac{\mathrm{A} \tau \sqrt{\left(\mathrm{C}_{55}-\rho \tau^{2}\right) / \mathrm{C}_{44}}}{(\tau-\beta) \sqrt{(\mathrm{x}-\mathrm{Vt}) \mathrm{x}}} \\
& \mathrm{w}=\mathrm{w}^{0}=\frac{1}{\mathrm{C}_{44}} \operatorname{Re} \int_{\mu}^{\mathrm{x} / \mathrm{t}} \frac{\mathrm{A} \tau}{(\tau-\beta) \sqrt{(\mathrm{V}-\tau) \tau}} \mathrm{d} \tau=\frac{1}{\mathrm{C}_{44}} \\
& \operatorname{Re} \int_{\mu}^{\mathrm{x} / \mathrm{t}}\left(1+\frac{\beta}{\tau-\beta}\right) \frac{\mathrm{A}}{\sqrt{(\mathrm{V}-\tau) \tau}} \mathrm{d} \tau \\
& =\frac{\mathrm{A}}{\mathrm{C}_{44}} \cdot \operatorname{Re}[2 \cdot \arcsin \\
& \sqrt{\frac{\tau}{\mathrm{V}}}+\frac{\beta}{\sqrt{1}} \ln \left|\frac{-\sqrt{(\mathrm{V}-\tau) \tau}+\sqrt{1}}{\tau-\beta}+\frac{\mathrm{V}+2 \beta}{2 \sqrt{1}}\right| \mid \infty \mathrm{x} \\
& =\frac{\mathrm{A}}{\mathrm{C}_{44}}\left|2 \cdot \arcsin \sqrt{\frac{\mathrm{x}}{\mathrm{Vt}}}+\frac{\beta}{\sqrt{1}} \ln \right| \begin{array}{l}
\frac{-\sqrt{(\mathrm{Vt}-\mathrm{x}) \mathrm{x}}+\mathrm{t} \sqrt{1}}{\mathrm{x}-\beta \mathrm{t}} \mid \\
+\frac{\mathrm{V}+2 \beta}{2 \sqrt{1}}
\end{array} \mid, \\
& 0<\mathrm{x}<\mathrm{Vt}
\end{aligned}
$$

where, $1=V \beta-\beta^{2}$. Its result is acquired by application of relative formulas in literature $(\mathrm{EGMH}, 2002)$ :

$$
\begin{aligned}
& \mathrm{K}_{3}(\mathrm{t})=\lim _{\mathrm{x} \rightarrow \mathrm{Vt}} \sqrt{2 \pi(\mathrm{x}-\mathrm{Vt})} \\
& \times \frac{\mathrm{A} \tau \sqrt{\left(\mathrm{C}_{55}-\rho \tau^{2}\right) / \mathrm{C}_{44}}}{(\tau-\beta) \sqrt{(\mathrm{x}-\mathrm{Vt}) \mathrm{x}}}=\frac{\mathrm{A} \sqrt{2 \pi \mathrm{V}\left(\mathrm{C}_{55}-\rho \mathrm{V}^{2}\right) / \mathrm{C}_{44}}}{(\mathrm{~V}-\beta) \sqrt{\mathrm{t}}}
\end{aligned}
$$

Known from Equation 23, stress intensity factor $\mathrm{K}_{3}(\mathrm{t})$ decays slowly and has obvious singularity on account of only one variable $t$ in the denominator and the rest quantities are regarded as real constants.

With all conditions remaining the same as that considered in the previous example, the loads become $\mathrm{Pt}^{2} / \mathrm{x}$. The boundary conditions will be as follows: 


$$
\begin{aligned}
& \tau_{\mathrm{yz}}(\mathrm{x}, 0, \mathrm{t})=-\mathrm{Pt}^{2} / \mathrm{x} \cdot \delta(\mathrm{x}-\beta \mathrm{t}), 0<\tau<\mathrm{V} \\
& \mathrm{w}(\mathrm{x}, 0, \mathrm{t})=0, \\
& \mathrm{x}>\mathrm{Vt}
\end{aligned}
$$

Apparently the stress will be homogeneous functions in which $L=1$. According to Equation 7 and 8 as well as the theory of generalized functions (Hoskins, 1979; Gahov, 1966), the first of Equation 24 can be rewritten as Equation 25:

$$
\operatorname{Re}[\mathrm{T}(\tau) \mathrm{f}(\tau)]=-(2 \mathrm{Pt} / \mathrm{x}) \mathrm{t} \delta(\mathrm{x}-\beta \mathrm{t})=-2 \mathrm{P} \tau^{-1} \delta(\tau-\beta), 0<\tau<\mathrm{V}
$$

At $x \neq \beta \mathrm{t}$, the derivative of $\delta(x-\beta t)$ is zero (Hoskins, $1979)$, then the result will be obtained.

Deducing from the above formulas, the unique solution of $f(\tau)$ must have this format:

$$
f(\tau)=\xi(\tau) /[\tau(\tau-\beta)]
$$

In the formula $\xi(\tau)$ has no singularity in the domain of $0<\tau<V$, while $T(\tau)$ is purely imaginary for the subsonic speeds; therefore $\xi(\tau)$ must be purely real in the segment of $0<\tau<\mathrm{V}$. Thus, Equation 26 induces Equation 27:

$$
\operatorname{Re} \xi(\tau)=0, \tau>\mathrm{V}, \operatorname{Im} \xi(\tau)=0,0<\tau<\mathrm{V}
$$

In the light of asymmetry and the conditions of the infinite point of the plane corresponding to the origin of coordinates of the physical plane as well as singularities of the crack tip (Gahov, 1966; Sih, 1977b; Kanwal and Sharma, 1976), the unique solution of the Keldysh-Sedov problem (27) can be obtained:

$$
\xi(\tau)=\frac{\mathrm{A}}{[(\mathrm{V}-\tau) \tau]^{3 / 2}}
$$

where, is an unbeknown constant.

Replacing Equation 28 into 26, one can gain:

$$
f(\tau)=\frac{A}{\tau(\tau-\beta)[(V-\tau) \tau]^{3 / 2}}
$$

Putting Equation 29 and 5 into 26, at $\tau \rightarrow \beta$, constant A will be determined as Equation 30:

$$
A=-\frac{2 P[(V-\beta) \beta]^{3 / 2}}{\pi \sqrt{\left(C_{55}-\rho \beta^{2}\right) / C_{44}}}
$$

In an orthotropic isotropic body, the disturbance range of elastic wave can be denoted by the circular region of radius $c_{1} t$ and $c_{2} t$, here $c_{1}$ and $c_{2}$ are the velocities of longitudinal and transverse waves $\left(c_{1}>c_{2}\right)$ of elastic body respectively. In an orthotropic anisotropic body, the disturbance range of elastic wave is not the circular area and can not exceed threshold value $\mathrm{C}_{\mathrm{d}}=\sqrt{\mathrm{C}_{55} / \rho}$ (sonic velocity) of elastic body. At $|\mathrm{x}|>\mathrm{C}_{\mathrm{d}} \mathrm{t}$, with $\operatorname{Im}[\mathrm{T}(\tau)]=0$, thus the stresses and the displacements are zero, which are accordant with the initial conditions; and this narrates that at the surface $\mathrm{y}=0$, disturbance range of elastic wave cannot overrun $\mathrm{C}_{\mathrm{d}} \mathrm{t}$.

Then putting Equation 29 and 5 into Equation 7 and 8 , at the surface $\mathrm{y}=0$, the stress $\tau_{\mathrm{yz}}$ and dynamic stress intensity factor $\mathrm{K}_{3}(\mathrm{t})$ are acquired, respectively Equation 31 and 32:

$$
\begin{aligned}
& \tau_{y z}=\int_{\infty}^{x} \frac{1}{t} \operatorname{Re} \frac{A \sqrt{\left(C_{55}-\rho \tau^{2}\right) / C_{44}}}{\tau(\tau-\beta)[(V-\tau) \tau]^{3 / 2}} d t \\
& \left.=-\operatorname{Re} \int_{C_{d}}^{\frac{x}{t}} \frac{A \sqrt{\left(C_{55}-\rho \tau^{2}\right) / C_{44}}}{\tau^{2}(\tau-\beta)[(\tau-V) \tau]^{3 / 2}} d \tau, x\right) \\
& \mathrm{K}_{3}(\mathrm{t})=\lim _{\mathrm{x} \rightarrow \mathrm{Vt}} \sqrt{2 \pi(\mathrm{x}-\mathrm{Vt})} \cdot \operatorname{Re} \int_{\mathrm{C}_{\mathrm{d}}}^{\frac{\mathrm{x}}{\mathrm{t}}}-\frac{\mathrm{A} \sqrt{\left(\mathrm{C}_{55}-\rho \tau^{2}\right) / \mathrm{C}_{44}}}{\tau^{2}(\tau-\beta)[(\tau-\mathrm{V}) \tau]^{3 / 2}} \mathrm{~d} \tau \\
& =2 \sqrt{2 \pi \mathrm{t}} \cdot \frac{\mathrm{A} \sqrt{\left(\mathrm{C}_{55}-\rho \mathrm{V}^{2}\right) / \mathrm{C}_{44}}}{(\mathrm{~V}-\beta) \mathrm{V}^{7 / 2}}
\end{aligned}
$$

The limit of above formula remains with the format of $0 . \infty$, which should be translated into the shape of $\infty / \infty$, then its result can be worked out by means of L'Hospital theorem (TOMTU, 1994). Dynamic stress intensity factor $\mathrm{K}_{3}(\mathrm{t})$ gradually increases from zero and even reaches or exceeds fracture toughness of this material with the time long, because variable $t$ lies in the numerator of this expression and the rest quantities are also referred to as real constants.

In order to show expediently, Equation 29 can be rewritten as:

$$
f(\tau)=\frac{A}{\tau(\tau-\beta)[(V-\tau) \tau]^{3 / 2}}=\frac{1}{\beta}\left(\frac{1}{\tau-\beta}-\frac{1}{\tau}\right) \frac{A}{[(V-\tau) \tau]^{3 / 2}}
$$

For the sake of convenience, it is assumed that:

$$
\mathrm{X}=(\mathrm{V}-\tau) \tau=\mathrm{V} \tau-\tau^{2}
$$

Based on the above results, the correlative constants of Equation (34) can be presented (EGMH, 2002) as: $\mathrm{a}=$ $0, \mathrm{~b}=\mathrm{V}, \mathrm{c}=-1, \mathrm{~K}=4 \mathrm{ac}-\mathrm{b}_{2}=-\mathrm{V}^{2}$

Integrating Equation 33 according to Equation 8, one will gain $\mathrm{w}^{0}$ Equation 35 : 
$\mathrm{w}^{0}=\frac{1}{\mathrm{C}_{44}} \int \mathrm{f}(\tau) \mathrm{d} \tau$

$=\frac{1}{C_{44}} \int \frac{A}{\tau(\tau-\beta) X^{3 / 2}} d \tau=\frac{-A}{C_{44} \beta} \int\left[\frac{1}{\tau}-\frac{1}{(\tau-\beta)}\right] \frac{d \tau}{X^{3 / 2}}$

But $\mathrm{w}^{0}$ will be obtained with two terms. After separating denotation and using the integral formulae in literature (EGMH, 2002), it has Equation 36:

$$
\begin{aligned}
& \mathrm{w}_{1}{ }^{0}=\frac{1}{\mathrm{C}_{44}} \int \mathrm{f}_{1}(\tau) \mathrm{d} \tau=-\frac{\mathrm{A}}{\mathrm{C}_{44} \beta} \int \frac{1}{\tau \cdot \mathrm{X}^{3 / 2}} \mathrm{~d} \tau \\
& =\frac{-2 \mathrm{~A}}{3 \beta \mathrm{C}_{44} \mathrm{~V}^{3}}\left[\frac{\mathrm{V}^{2}}{\tau \sqrt{\mathrm{X}}}-\frac{4 \sqrt{\mathrm{X}}}{\tau}+\frac{4 \tau}{\sqrt{\mathrm{X}}}\right]+\mathrm{C} \\
& \mathrm{w}_{2}{ }^{0}=\frac{1}{\mathrm{C}_{44}} \int \mathrm{f}_{2}(\tau) \mathrm{d} \tau=\frac{\mathrm{A}}{\mathrm{C}_{44} \beta} \int \frac{1}{(\tau-\beta)} \frac{\mathrm{d} \tau}{\mathrm{X}^{3 / 2}}
\end{aligned}
$$

Denominator in Equation 37 contains this term $(\tau$ $\beta) \mathrm{X}^{3 / 2}$, therefore the computation is incapable of using directly integral formulas, thus integral format must translate into performable integral (EGMH, 2002).

By variable substitution: $\tau_{1}=\tau-\beta$, now putting it into Equation 34, one can acquire:

$$
\mathrm{X}=(\mathrm{V}-\tau) \tau=\mathrm{V} \beta-\beta^{2}+(\mathrm{V}-2 \beta) \tau_{1}-\tau_{1}^{2}
$$

The correlative constants of Equation 38 can be denoted as follows: $a_{1}=V \beta-\beta^{2}, b_{1}=V-2 \beta, c=-1$, $\mathrm{K}_{1}=4 \mathrm{a}_{1} \mathrm{c}-1^{2}=-\mathrm{V}^{2}=\mathrm{K}$.

Integrating Equation 37 by means of variable substitution, $w_{2}^{0}$ can be rewritten $(\mathrm{EGMH}, 2002)$ as:

$$
\begin{aligned}
& \mathrm{w}_{2}{ }^{0}=\frac{\mathrm{A}}{\mathrm{C}_{44} \beta} \int \frac{\mathrm{d} \tau}{(\tau-\beta) \mathrm{X}^{3 / 2}}=\frac{\mathrm{A}}{\beta \mathrm{C}_{44}} \int \frac{\mathrm{d} \tau_{1}}{\tau_{1} \mathrm{X}^{3 / 2}} \\
& =\frac{\mathrm{A}}{\mathrm{a}_{1} \beta \mathrm{C}_{44}}\left[\frac{1}{\sqrt{\mathrm{X}}}+\int \frac{\mathrm{d} \tau_{1}}{\tau_{1} \sqrt{\mathrm{X}}}-\frac{\mathrm{b}_{1}}{2} \int \frac{\mathrm{d} \tau_{1}}{\mathrm{X}^{3 / 2}}\right] \\
& =\frac{\mathrm{A}}{\mathrm{a}_{1} \beta \mathrm{C}_{44}}\left[\frac{1}{\sqrt{\mathrm{X}}}-\frac{1}{\sqrt{\mathrm{a}_{1}}} \ln \left|\frac{\sqrt{\mathrm{X}}+\sqrt{\mathrm{a}_{1}}}{\tau_{1}}+\frac{\mathrm{b}_{1}}{2 \sqrt{\mathrm{a}_{1}}}\right|\right. \\
& \left.-\frac{\mathrm{b}_{1}}{2} \cdot \frac{-4 \tau_{1}+2 \mathrm{~b}_{1}}{\mathrm{~K} \sqrt{\mathrm{X}}}\right]=\frac{\mathrm{A}}{\mathrm{a}_{1} \beta \mathrm{C}_{44}}\left[\frac{\mathrm{K}-\mathrm{b}_{1}^{2}}{\mathrm{~K} \sqrt{\mathrm{X}}}-\frac{1}{\sqrt{\mathrm{a}_{1}}}\right. \\
& \left.\ln \left|\frac{\sqrt{\mathrm{X}}+\sqrt{\mathrm{a}_{1}}}{\tau_{1}}+\frac{\mathrm{b}_{1}}{2 \sqrt{\mathrm{a}_{1}}}\right|+\frac{2 \mathrm{~b}_{1} \tau_{1}}{\mathrm{~K} \sqrt{\mathrm{X}}}\right]=\frac{\mathrm{A}}{\mathrm{a}_{1} \beta \mathrm{C}_{44}}\left[\frac{\mathrm{K}-\mathrm{b}_{1}^{2}-2 \mathrm{~b}_{1} \beta}{\mathrm{K} \sqrt{\mathrm{X}}}\right. \\
& \left.-\frac{1}{\sqrt{\mathrm{a}_{1}}} \ln \left|\frac{\sqrt{\mathrm{X}}+\sqrt{\mathrm{a}_{1}}}{\tau-\beta}+\frac{\mathrm{b}_{1}}{2 \sqrt{\mathrm{a}_{1}}}\right|+\frac{2 \mathrm{~b}_{1} \tau}{\mathrm{K} \sqrt{\mathrm{X}}}\right]+\mathrm{C}
\end{aligned}
$$

Known from Equation 35: $\mathrm{w}^{0}(\tau)=\mathrm{w}_{1}{ }^{0}(\tau)+\mathrm{w}_{2}{ }^{0}(\tau)$. The crack runs along the $x$-axis, therefore $\mathrm{W}^{0}(\tau)$ comprising Equation 36 and 39 can be performed in the definite integral operation, one takes constant $\mathrm{C}=0$.

Now putting Equation 36 into 7, the divisional displacement $\mathrm{W}_{1}$ is Equation 40:

$$
\begin{aligned}
& \mathrm{W}_{1}=\int_{0}^{\mathrm{t}} \operatorname{Rew}_{1}^{0}(\tau) \mathrm{dt}=\operatorname{Re} \int_{\infty}^{\mathrm{x} / \mathrm{t}}-\frac{\mathrm{x}}{\tau^{2}} \mathrm{w}_{1}^{0}(\tau) \mathrm{d} \tau \\
& =\frac{2 \mathrm{~A}}{3 \beta \mathrm{C}_{44} \mathrm{~V}^{3}} \operatorname{Re} \int_{\infty}^{\mathrm{x} / \mathrm{t}} \frac{\mathrm{x}}{\tau^{2}}\left[\frac{\mathrm{V}^{2}}{\tau \sqrt{\mathrm{X}}}-\frac{4 \sqrt{\mathrm{X}}}{\tau}+\frac{4 \tau}{\sqrt{\mathrm{X}}}\right] \mathrm{d} \tau
\end{aligned}
$$

Applying integral formulas in literature (EGMH, 2002), the following representations are attained:

$$
\mathrm{S}_{1}=\int \frac{\mathrm{d} \tau}{\tau \sqrt{\mathrm{X}}}=-\frac{2 \sqrt{\mathrm{X}}}{\mathrm{b} \tau}=-\frac{2}{\mathrm{~b}} \sqrt{\frac{\mathrm{V}-\tau}{\tau}},(\text { for } \mathrm{a}=0)
$$

$$
\begin{aligned}
& S_{2}=\int \frac{d \tau}{\tau^{2} \sqrt{X}} \\
& =\frac{-2}{V^{2}} \sqrt{\frac{V-\tau}{\tau}}\left(1+\frac{V-\tau}{3 \tau}\right)=-\frac{2}{V^{2}} \frac{\sqrt{X}}{\tau}\left(1+\frac{X}{3 \tau^{2}}\right),(\text { for } \mathrm{a}=0)
\end{aligned}
$$

$$
\begin{aligned}
& \mathrm{S}_{3}=\int \frac{\mathrm{d} \tau}{\tau^{3} \sqrt{\mathrm{X}}}=-\frac{2}{\mathrm{~V}^{3}} \sqrt{\frac{\mathrm{V}-\tau}{\tau}}\left[\frac{1}{5}\left(\sqrt{\frac{\mathrm{V}-\tau}{\tau}}\right)^{4}\right. \\
& \left.+\frac{2}{3}\left(\sqrt{\frac{\mathrm{V}-\tau}{\tau}}\right)^{2}+1\right]=-\frac{2}{\mathrm{~V}^{3}} \frac{\sqrt{\mathrm{X}}}{\tau}\left(\frac{1}{5} \cdot \frac{\mathrm{X}^{2}}{\tau^{4}}+\frac{2 \mathrm{X}}{3} \frac{\mathrm{X}}{\tau^{2}}+1\right),(\text { for } \mathrm{a}=0)
\end{aligned}
$$

Equation 41, 42 and 43 are deducted by this relationship: $\tau=V \sin ^{2} \theta$.

After putting Equation 41, 42 and 43 into 40, the divisional displacement $\mathrm{w}_{1}$ is acquired Equation 44 : 


$$
\begin{aligned}
& \mathrm{w}_{1}=\frac{2 \mathrm{Ax}}{3 \beta \mathrm{C}_{44} \mathrm{~V}^{3}} \operatorname{Re}\left[-\frac{2}{\mathrm{~V}} \frac{\sqrt{\mathrm{X}}}{\tau}\left(\frac{1}{5} \cdot \frac{\mathrm{X}^{2}}{\tau^{4}}+\frac{2}{3} \frac{\mathrm{X}}{\tau^{2}}+1\right)\right. \\
& -4\left(-\frac{\sqrt{\mathrm{X}}}{2 \tau^{2}}+\frac{\mathrm{b}}{4} \int \frac{1}{\tau^{2} \sqrt{\mathrm{X}}} \mathrm{d} \tau\right. \\
& \left.\left.-\frac{1}{2} \int \frac{1}{\tau \sqrt{\mathrm{X}}} \mathrm{d} \tau\right)-4 \infty \frac{2 \sqrt{\mathrm{X}}}{\mathrm{b} \tau}\right]\left.\right|_{\infty} ^{\mathrm{x} / \mathrm{t}}=\frac{2 \mathrm{Ax}}{3 \beta \mathrm{C}_{44} \mathrm{~V}^{3}} \\
& \operatorname{Re}\left[-\frac{2}{\mathrm{~V}} \frac{\sqrt{\mathrm{X}}}{\tau}\left(\frac{1}{5} \cdot \frac{\mathrm{X}^{2}}{\tau^{4}}+\frac{2}{3} \frac{\mathrm{X}}{\tau^{2}}+1\right)+\frac{2 \sqrt{\mathrm{X}}}{\tau^{2}}\right. \\
& \left.+\mathrm{b} \cdot \frac{2}{\mathrm{~V}^{2}} \frac{\sqrt{\mathrm{X}}}{\tau}\left(1+\frac{1}{3} \cdot \frac{\mathrm{X}}{\tau^{2}}\right)-\frac{4 \sqrt{\mathrm{X}}}{\mathrm{b} \tau}-\frac{8 \sqrt{\mathrm{X}}}{\mathrm{b} \tau}\right]\left.\right|_{\infty} ^{\mathrm{x} / \mathrm{t}} \\
& =\frac{2 \mathrm{Ax}}{3 \beta \mathrm{C}_{44} \mathrm{~V}^{3}} \operatorname{Re}\left[-\frac{2}{5 \mathrm{~V}} \cdot \frac{\mathrm{X}^{5 / 2}}{\tau^{5}}-\frac{4}{3 \mathrm{~V}} \cdot \frac{\mathrm{X}^{3 / 2}}{\tau^{3}}\right. \\
& \left.-\frac{2 \sqrt{\mathrm{X}}}{\mathrm{V} \tau}+\frac{2 \sqrt{\mathrm{X}}}{\tau^{2}}++\frac{2 \mathrm{~b}}{\mathrm{~V}^{2}} \frac{\sqrt{\mathrm{X}}}{\tau}+\frac{2 \mathrm{~b}}{3 \mathrm{~V}^{2}} \cdot \frac{\mathrm{X}^{3 / 2}}{\tau^{3}}-\frac{12 \sqrt{\mathrm{X}}}{\mathrm{b} \tau}\right]\left.\right|_{\infty} ^{\mathrm{x} / \mathrm{t}} \\
& =\frac{2 \mathrm{Ax}}{3 \beta \mathrm{C}_{44} \mathrm{~V}^{3}} \operatorname{Re}\left[-\frac{2}{5 \mathrm{~V}} \cdot \frac{\mathrm{X}^{5 / 2}}{\tau^{5}}+\left(\frac{2 \mathrm{~b}}{3 \mathrm{~V}^{2}}-\frac{4}{3 \mathrm{~V}}\right) \frac{\mathrm{X}^{3 / 2}}{\tau^{3}}\right. \\
& \left.+\left(\frac{2 \mathrm{~b}}{\mathrm{~V}^{2}}-\frac{2}{\mathrm{~V}}-\frac{12}{\mathrm{~b}}\right) \frac{\sqrt{\mathrm{X}}}{\tau}+\frac{2 \sqrt{\mathrm{X}}}{\tau^{2}}\right]\left.\right|_{\infty} ^{\mathrm{x} / \mathrm{t}} \\
& =\left.\frac{2 \mathrm{Ax}}{3 \beta \mathrm{C}_{44} \mathrm{~V}^{3}} \operatorname{Re}\left[-\frac{2}{5 \mathrm{~V}} \cdot \frac{\mathrm{X}^{5 / 2}}{\tau^{5}}-\frac{2}{3 \mathrm{~V}} \cdot \frac{\mathrm{X}^{3 / 2}}{\tau^{3}}-\frac{12}{\mathrm{~b}} \cdot \frac{\sqrt{\mathrm{X}}}{\tau}+\frac{2 \sqrt{\mathrm{X}}}{\tau^{2}}\right]\right|_{\infty} ^{\mathrm{x} / \mathrm{t}} \\
& =\frac{2 \mathrm{Ax}}{3 \beta \mathrm{C}_{44} \mathrm{~V}^{3}} \operatorname{Re}\left[-\frac{2}{5 \mathrm{~V}} \cdot\left(\frac{\mathrm{V}-\tau}{\tau}\right)^{5 / 2}-\frac{2}{3 \mathrm{~V}} \cdot\left(\frac{\mathrm{V}-\tau}{\tau}\right)^{3 / 2}\right. \\
& \left.-\frac{12}{\mathrm{~b}} \cdot\left(\frac{\mathrm{V}-\tau}{\tau}\right)^{1 / 2}+\frac{2 \sqrt{\mathrm{V}-\tau}}{\tau^{3 / 2}}\right]\left.\right|_{\infty} ^{\mathrm{x} / \mathrm{t}} \\
& =\frac{2 \mathrm{Ax}}{3 \beta \mathrm{C}_{44} \mathrm{~V}^{3}}\left\{\left[-\frac{2}{5 \mathrm{~V}} \cdot\left(\frac{\mathrm{Vt}-\mathrm{x}}{\mathrm{x}}\right)^{2}\right.\right. \\
& \left.\left.-\frac{2}{3 \mathrm{~V}} \cdot \frac{\mathrm{Vt}-\mathrm{x}}{\mathrm{x}}-\frac{12}{\mathrm{~b}}\right] \sqrt{\frac{\mathrm{Vt}-\mathrm{x}}{\mathrm{x}}}+\frac{2 \mathrm{t} \sqrt{\mathrm{Vt}-\mathrm{x}}}{\mathrm{x}^{3 / 2}}\right\}\left.\right|_{\infty} ^{\mathrm{x} / \mathrm{t}} \\
& =\frac{2 \mathrm{~A}}{3 \beta \mathrm{C}_{44} \mathrm{~V}^{3}}\left\{\left[-\frac{2}{5 \mathrm{~V}} \cdot\left(\frac{\mathrm{Vt}-\mathrm{x}}{\mathrm{x}}\right)^{2}-\frac{2}{3 \mathrm{~V}} \cdot \frac{\mathrm{Vt}-\mathrm{x}}{\mathrm{x}}-\frac{12}{\mathrm{~b}}\right]\right. \\
& \left.\sqrt{(\mathrm{Vt}-\mathrm{x}) \mathrm{x}}+2 \mathrm{t} \sqrt{\frac{\mathrm{Vt}-\mathrm{x}}{\mathrm{x}}}\right\}, 0<\mathrm{x}<\mathrm{Vt}
\end{aligned}
$$

Then replacing Equation 39 into 7, the separated displacement $\mathrm{W}_{2}$ is as:

$$
\begin{aligned}
& \mathrm{w}_{2}=\int_{0}^{\mathrm{t}} \operatorname{Rew}_{2}^{0}(\tau) \mathrm{dt}=\operatorname{Re} \int_{\infty}^{\mathrm{x} / \mathrm{t}}-\frac{\mathrm{x}}{\tau^{2}} \mathrm{w}_{2}^{0}(\tau) \mathrm{d} \tau \\
& =-\frac{\mathrm{Ax}}{\mathrm{C}_{44} \mathrm{a}_{1} \beta} \operatorname{Re} \int_{\infty}^{\mathrm{x} / \mathrm{t}} \frac{1}{\tau^{2}}\left[\frac{\mathrm{K}-\mathrm{b}_{1}^{2}-2 \mathrm{~b}_{1} \beta}{\mathrm{K} \sqrt{\mathrm{X}}}\right. \\
& \left.-\frac{1}{\sqrt{\mathrm{a}_{1}}} \ln \left|\frac{\sqrt{\mathrm{X}}+\sqrt{\mathrm{a}_{1}}}{\tau-\beta}+\frac{\mathrm{b}_{1}}{2 \sqrt{\mathrm{a}_{1}}}\right|+\frac{2 \mathrm{~b}_{1} \tau}{\mathrm{K} \sqrt{\mathrm{X}}}\right] \mathrm{d} \tau
\end{aligned}
$$

The integral of the second term of Equation 45 without coefficient can be written as follows:

$$
\begin{aligned}
& \int-\frac{1}{\tau^{2} \sqrt{\mathrm{a}_{1}}} \ln \left|\frac{\sqrt{\mathrm{X}}+\sqrt{\mathrm{a}_{1}}}{\tau-\beta}+\frac{\mathrm{b}_{1}}{2 \sqrt{\mathrm{a}_{1}}}\right| \mathrm{d} \tau=\frac{1}{\sqrt{\mathrm{a}_{1}}} \\
& \int \ln \left|\frac{\sqrt{\mathrm{X}}+\sqrt{\mathrm{a}_{1}}}{\tau-\beta}+\frac{\mathrm{b}_{1}}{2 \sqrt{\mathrm{a}_{1}}}\right| \mathrm{d}\left(\frac{1}{\tau}\right) \\
& =\frac{1}{\tau \sqrt{\mathrm{a}_{1}}} \ln \left|\frac{\sqrt{\mathrm{X}}+\sqrt{\mathrm{a}_{1}}}{\tau-\beta}+\frac{\mathrm{b}_{1}}{2 \sqrt{\mathrm{a}_{1}}}\right|-\frac{1}{\sqrt{\mathrm{a}_{1}}} \\
& \int \frac{1}{\tau} \cdot \frac{1}{\frac{\sqrt{\mathrm{X}}+\sqrt{\mathrm{a}_{1}}}{\tau-\beta}+\frac{\mathrm{b}_{1}}{2 \sqrt{\mathrm{a}_{1}}}} \\
& \times\left[\frac{\frac{\mathrm{b}-2 \tau}{2 \sqrt{\mathrm{X}}} \cdot(\tau-\beta)-\left(\sqrt{\mathrm{X}}+\sqrt{\mathrm{a}_{1}}\right)}{(\tau-\beta)^{2}}\right] \mathrm{d} \tau
\end{aligned}
$$




$$
\begin{aligned}
& =\frac{1}{\tau{\sqrt{\mathrm{a}_{1}}}_{1}} \ln \left|\frac{\sqrt{\mathrm{X}}+\sqrt{\mathrm{a}_{1}}}{\tau-\beta}+\frac{\mathrm{b}_{1}}{2 \sqrt{\mathrm{a}_{1}}}\right| \\
& -\frac{1}{\sqrt{\mathrm{a}_{1}}} \int \frac{1}{\tau} \cdot \frac{2 \sqrt{\mathrm{a}_{1}}(\tau-\beta)}{2 \sqrt{\mathrm{a}_{1} \mathrm{X}}+2 \mathrm{a}_{1}+\mathrm{b}_{1}(\tau-\beta)} . \\
& \frac{(b-2 \tau)(\tau-\beta)-2 X-2 \sqrt{a_{1} X}}{2 \sqrt{X} \cdot(\tau-\beta)^{2}} \cdot d \tau \\
& =\frac{1}{\tau \sqrt{\mathrm{a}_{1}}} \ln \left|\frac{\sqrt{\mathrm{X}}+\sqrt{\mathrm{a}_{1}}}{\tau-\beta}+\frac{\mathrm{b}_{1}}{2 \sqrt{\mathrm{a}_{1}}}\right|- \\
& \int_{\tau}^{1} \frac{-b \beta-b \tau-2 a+2 \tau \beta-2 \sqrt{a_{1} X}}{2 \sqrt{a_{1} X}+2 a_{1}+b_{1}(\tau-\beta)} \cdot \frac{1}{\sqrt{X} \cdot(\tau-\beta)} d \tau \\
& =\frac{1}{\tau \sqrt{\mathrm{a}_{1}}} \ln \left|\frac{\sqrt{\mathrm{X}}+\sqrt{\mathrm{a}_{1}}}{\tau-\beta}+\frac{\mathrm{b}_{1}}{2 \sqrt{\mathrm{a}_{1}}}\right|- \\
& \int \frac{1}{\tau} \cdot \frac{-b \beta-b \tau-2 a+2 \tau \beta-2 \sqrt{a_{1} X}}{2 \sqrt{a_{1} X}+2\left(a+b \beta-\beta^{2}\right)+(b-2 \beta)(\tau-\beta)} \cdot \frac{1}{\sqrt{X}(\tau-\beta)} d \tau \\
& =\frac{1}{\tau \sqrt{\mathrm{a}_{1}}} \ln \left|\frac{\sqrt{\mathrm{X}}+\sqrt{\mathrm{a}_{1}}}{\tau-\beta}+\frac{\mathrm{b}_{1}}{2 \sqrt{\mathrm{a}_{1}}}\right|+ \\
& \int \frac{1}{\tau} \cdot \frac{\mathrm{d} \tau}{(\tau-\beta) \sqrt{\mathrm{X}}}=\frac{1}{\tau \sqrt{\mathrm{a}_{1}}} \ln \left|\frac{\sqrt{\mathrm{X}}+\sqrt{\mathrm{a}_{1}}}{\tau-\beta}+\frac{\mathrm{b}_{1}}{2 \sqrt{\mathrm{a}_{1}}}\right| \\
& +\frac{1}{\beta}\left(\frac{2 \sqrt{\mathrm{X}}}{\mathrm{b} \tau}+\frac{1}{\sqrt{\mathrm{a}_{1}}} \ln \mid \frac{\sqrt{\mathrm{a}_{1}}-\sqrt{\mathrm{X}}}{\tau-\beta}+\frac{\mathrm{b}_{1}}{2 \sqrt{\mathrm{a}_{1}}}\right)
\end{aligned}
$$

Then inserting Equation 46, 41 and 42 into 45, the zonal displacement $\mathrm{W}_{2}$ is:

$$
\begin{aligned}
& \mathrm{w}_{2}=-\frac{\mathrm{Ax}}{\mathrm{C}_{44} \mathrm{a}_{1} \beta} \operatorname{Re}\left\{\frac{\mathrm{K}-\mathrm{b}_{1}^{2}-2 \mathrm{~b}_{1} \beta}{\mathrm{K}} \cdot \mathrm{S}_{2}+\frac{2 \mathrm{~b}_{1}}{\mathrm{~K}} \mathrm{~S}_{1}\right. \\
& +\frac{1}{\tau \sqrt{\mathrm{a}_{1}}} \ln \left|\frac{\sqrt{\mathrm{X}}+\sqrt{\mathrm{a}_{1}}}{\tau-\beta}+\frac{\mathrm{b}_{1}}{2 \sqrt{\mathrm{a}_{1}}}\right|+\frac{1}{\beta}\left[-\mathrm{S}_{1}+\frac{1}{\sqrt{\mathrm{a}_{1}}}\right. \\
& \left.\left.\ln \mid \frac{\sqrt{\mathrm{a}_{1}}-\sqrt{\mathrm{X}}}{\tau-\beta}+\frac{\mathrm{b}_{1}}{2 \sqrt{\mathrm{a}_{1}}}\right]\right\}||_{\infty}^{\mathrm{x} / \mathrm{t}}=\frac{\mathrm{A}}{\mathrm{C}_{44} \mathrm{a}_{1} \beta} \operatorname{Re}\left\{\frac{\mathrm{K}-\mathrm{b}_{1}^{2}-2 \mathrm{~b}_{1} \beta}{\mathrm{K}}\right. \\
& \frac{2}{3 \mathrm{~V}^{2}} \sqrt{\frac{\mathrm{V}-\tau}{\tau}}(2 \mathrm{x}+\mathrm{Vt})-\frac{2 \mathrm{t} \sqrt{\mathrm{X}}}{\mathrm{b}}\left(-\frac{2 \mathrm{~b}_{1}}{\mathrm{~K}}+\frac{1}{\beta}\right)-\frac{\mathrm{t}}{\sqrt{\mathrm{a}_{1}}} \\
& \left.\ln \left|\frac{\sqrt{\mathrm{a}_{1}}+\sqrt{\mathrm{X}}}{\tau-\beta}+\frac{\mathrm{b}_{1}}{2 \sqrt{\mathrm{a}_{1}}}\right|-\frac{\mathrm{x}}{\beta \sqrt{\mathrm{a}_{1}}} \ln \left|\frac{\sqrt{\mathrm{a}_{1}}-\sqrt{\mathrm{X}}}{\tau-\beta}+\frac{\mathrm{b}_{1}}{2 \sqrt{\mathrm{a}_{1}}}\right|\right\} \mid \mathrm{x} / \mathrm{t} \\
& =\frac{\mathrm{A}}{\mathrm{C}_{44} \mathrm{a}_{1} \beta}\left\{\frac{\mathrm{K}-\mathrm{b}_{1}^{2}-2 \mathrm{~b}_{1} \beta}{\mathrm{K}} \times \frac{2}{3 \mathrm{~V}^{2}} \sqrt{\frac{\mathrm{Vt}-\mathrm{x}}{\mathrm{x}}}(2 \mathrm{x}+\mathrm{Vt})\right. \\
& -\frac{2 \sqrt{(V t-x) x}}{b}\left(-\frac{2 b_{1}}{K}+\frac{1}{\beta}\right)-\frac{t}{\sqrt{a_{1}}} \ln \left|\frac{t \sqrt{a_{1}}+\sqrt{(V t-x) x}}{x-\beta t}+\frac{b_{1}}{2 \sqrt{a_{1}}}\right| \\
& \left.-\frac{\mathrm{x}}{\beta \sqrt{\mathrm{a}_{1}}} \ln \left|\frac{\mathrm{t} \sqrt{\mathrm{a}_{1}}-\sqrt{(\mathrm{Vt}-\mathrm{x}) \mathrm{x}}}{\mathrm{x}-\beta \mathrm{t}}+\frac{\mathrm{b}_{1}}{2 \sqrt{\mathrm{a}_{1}}}\right|\right\} 0<\mathrm{x}<\mathrm{Vt}
\end{aligned}
$$

The displacement is the summation of subdistrict displacement: $\mathrm{W}=\mathrm{W}_{1}+\mathrm{W}_{2}$. After the addition between Equation 44 and 47, the displacement $\mathrm{W}$ is attained Equation 48:

$$
\begin{aligned}
& \mathrm{w}=\frac{2 \mathrm{~A}}{3 \beta \mathrm{C}_{44} \mathrm{~V}^{3}}\left\{\left[-\frac{2}{5 \mathrm{~V}} \cdot\left(\frac{\mathrm{Vt}-\mathrm{x}}{\mathrm{x}}\right)^{2}-\right.\right. \\
& \left.\left.\frac{2}{3 \mathrm{~V}} \cdot \frac{\mathrm{Vt}-\mathrm{x}}{\mathrm{x}}-\frac{12}{\mathrm{~b}}\right] \sqrt{(\mathrm{Vt}-\mathrm{x}) \mathrm{x}}+2 \mathrm{t} \sqrt{\frac{\mathrm{Vt}-\mathrm{x}}{\mathrm{x}}}\right\} \\
& +\frac{\mathrm{A}}{\beta \mathrm{a}_{1} \mathrm{C}_{44}}\left\{\frac{\mathrm{K}-\mathrm{b}_{1}^{2}-2 \mathrm{~b}_{1} \beta}{\mathrm{K}} \cdot \frac{2}{3 \mathrm{~V}^{2}}\right. \\
& \sqrt{\frac{V t-x}{x}}(2 x+V t)-\frac{2 \sqrt{(V t-x) x}}{b}\left(-\frac{2 b_{1}}{K}+\frac{1}{\beta}\right) \\
& -\frac{\mathrm{t}}{\sqrt{\mathrm{a}_{1}}} \ln \left|\frac{\mathrm{t} \sqrt{\mathrm{a}_{1}}+\sqrt{(\mathrm{Vt}-\mathrm{x}) \mathrm{x}}}{\mathrm{x}-\beta \mathrm{t}}+\frac{\mathrm{b}_{1}}{2 \sqrt{\mathrm{a}_{1}}}\right| \\
& \left.-\frac{\mathrm{x}}{\beta \sqrt{\mathrm{a}_{1}}} \ln \left|\frac{\mathrm{t} \sqrt{\mathrm{a}_{1}}-\sqrt{(\mathrm{Vt}-\mathrm{x}) \mathrm{x}}}{\mathrm{x}-\beta \mathrm{t}}+\frac{\mathrm{b}_{1}}{2 \sqrt{\mathrm{a}_{1}}}\right|\right\} \\
& =\frac{2 \mathrm{~A}}{\beta \mathrm{C}_{44}}\left\{\frac { 2 } { 3 \mathrm { V } ^ { 3 } } \left[-\frac{1}{5 \mathrm{~V}} \cdot\left(\frac{\mathrm{Vt}-\mathrm{x}}{\mathrm{x}}\right)^{2}-\right.\right. \\
& \left.\left.\frac{1}{3 \mathrm{~V}} \cdot \frac{\mathrm{Vt}-\mathrm{x}}{\mathrm{x}}-\frac{6}{\mathrm{~b}}\right]-\frac{1}{\mathrm{ba}_{1}}\left(\frac{1}{\beta}-\frac{2 \mathrm{~b}_{1}}{\mathrm{~K}}\right)\right\} \sqrt{(\mathrm{Vt}-\mathrm{x}) \mathrm{x}} \\
& -\frac{\mathrm{A}}{\beta \mathrm{a}_{1}{ }^{3 / 2} \mathrm{C}_{44}}\left[\mathrm{t} \ln \left|\frac{\mathrm{t} \sqrt{\mathrm{a}_{1}}+\sqrt{(\mathrm{Vt}-\mathrm{x}) \mathrm{x}}}{\mathrm{x}-\beta \mathrm{t}}+\frac{\mathrm{b}_{1}}{2 \sqrt{\mathrm{a}_{1}}}\right|\right. \\
& \left.+\frac{x}{\beta} \ln \left|\frac{t \sqrt{a_{1}}-\sqrt{(V t-x) x}}{x-\beta t}+\frac{b_{1}}{2 \sqrt{a_{1}}}\right|\right] \\
& +\frac{2 \mathrm{~A}}{3 \mathrm{~V}^{2} \beta \mathrm{C}_{44}}\left\{\left[\frac{\mathrm{K}-\mathrm{b}_{1}^{2}-2 \mathrm{~b}_{1} \beta}{\mathrm{a}_{1} \mathrm{~K}}\right.\right. \\
& \left.\left.(2 \mathrm{x}+\mathrm{Vt})+\frac{2 \mathrm{t}}{\mathrm{V}}\right]\right\} \sqrt{\frac{\mathrm{Vt}-\mathrm{x}}{\mathrm{x}}} 0<\mathrm{x}<\mathrm{Vt}
\end{aligned}
$$

where, $a=0, b=V, c=-1, K=4 a c-b^{2}=-V^{2}, a_{1}=V \beta$ $\beta^{2}, b_{1}=V-2 \beta, c=-1, K_{1}=4 a_{1} c-b_{1}^{2}=-V^{2}=K$

\subsection{Variational Law of Dynamic Stress Intensity Factor}

Analytical solutions need changing into numerical solutions according to practical situation of concrete problems, so variable rule of dynamic stress intensity factor can be depicted effectually. The corresponding parameters are put into Equation 23 and 32 to facilely plot $\mathrm{K}_{3}(\mathrm{t})$ as a function of time $\mathrm{t}$, respectively. The following constants (Wu, 2000; Nian-Chun et al., 2008; Lu et al., 2010a) presumed are as follows:

$\mathrm{C}_{44}=8 \mathrm{GPa}, \rho=2.7 \times 9.8 \times 10^{3} \mathrm{~N} \cdot \mathrm{m}^{-3} \mathrm{C}_{55}=16 \mathrm{GPa}$, $\mathrm{V}=300 \mathrm{~m} \cdot \mathrm{s}^{-1} ; \mathrm{P}=200 \mathrm{~N}, \beta=180 \mathrm{~m} \cdot \mathrm{s}^{-1}$ 


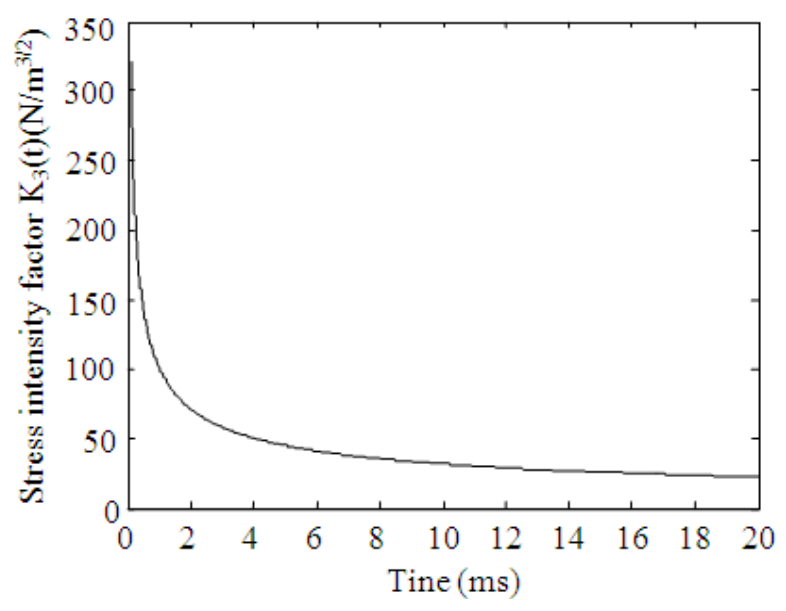

Fig. 2. Dynamic stress intensity factors $\mathrm{K}_{3}(\mathrm{t})$ versus time $\mathrm{t}$

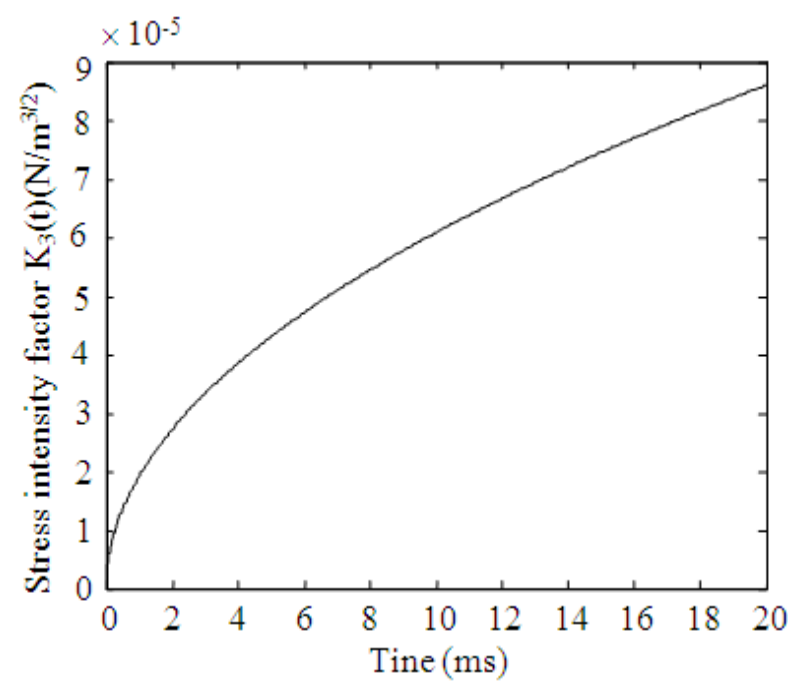

Fig. 3. Dynamic stress intensity factor $K_{3}(t)$ versus time $t$

Table 1. Relevant numerical values between dynamic stress intensity factor $\mathrm{K}_{3}(\mathrm{t})$ versus time $\mathrm{t}$

\begin{tabular}{lrrrrc}
\hline $\mathrm{t} / \mathrm{ms}$ & \multicolumn{1}{c}{2} & \multicolumn{1}{c}{3} & \multicolumn{1}{c}{4} & \multicolumn{1}{c}{5} \\
\hline $\mathrm{K}_{3}(\mathrm{t}) / \mathrm{N} . \mathrm{m}^{-3 / 2}$ & 71.784 & 50.759 & 41.444 & 35.892 & 32.103 \\
$\mathrm{t} / \mathrm{ms}$ & 6.000 & 7.000 & 8.000 & 9.000 & 10.000 \\
$\mathrm{~K}_{3}(\mathrm{t}) / \mathrm{N}^{-} \mathrm{m}^{-3 / 2}$ & 29.306 & 27.132 & 25.379 & 23.928 & 22.701 \\
\hline
\end{tabular}

Table 2. Relevant numerical values between dynamic stress intensity factor $\mathrm{K}_{3}(\mathrm{t})$ versus time $\mathrm{t}$

\begin{tabular}{lccccr}
\hline $\mathrm{t} / \mathrm{ms}$ & 1 & 2 & 3 & 4 & \multicolumn{1}{c}{5} \\
\hline $\mathrm{K}_{3}(\mathrm{t}) \times 10^{-5} / \mathrm{N} . \mathrm{m}^{-3 / 2}$ & 2.7181 & 3.8438 & 4.7077 & 5.4359 & 6.0776 \\
$\mathrm{t} / \mathrm{ms}$ & 6.0000 & 7.0000 & 8.0000 & 9.0000 & 10.0000 \\
$\mathrm{~K}_{3}(\mathrm{t}) \times 10^{-5} / \mathrm{N} \cdot \mathrm{m}^{-3 / 2}$ & 6.6576 & 7.1911 & 7.6876 & 8.1539 & 8.5953 \\
\hline
\end{tabular}

Known from Equation 23, dynamic stress intensity factor reduces tardily and trends to a constant finally and has obvious singularity with the increase of time in virtue of only one variable $t$ in the denominator, as displayed in Fig. 2. The correlative numerical value relationship is shown in Table 1. This changeable current is similar to the outcome in literatures (NianChun et al., 2008; Lu et al., 2010a; Kalthoff et al., 1977; Kobayashi, 1979), accordingly the obtained result is approved to be correct. In terms of Equation 32 , dynamic stress intensity factor laggardly increase from zero owing to variable $t$ in the numerator, but its increasing tend is slow and even reaches or exceeds fracture toughness of this material, hence structural destruction will occur, as shown in Fig. 3. Such a trend is homogeneous to the result in literatures (Nian-Chun et al., 2008; Lu et al., 2010a; RaviChandar and Knauss, 1984a; 1984b; 1984c; 1984d), so it is also right. The relative numerical value relation is indicated in Table 2 .

\section{CONCLUSION}

Using the relative expression: $f(x, y, t)=t^{n} f(x / t, y / t)$, just $\mathrm{n}$ is an integer number; and then the problem researched will be facilely translated into homogeneous functions of zeroth dimension, i.e., homogeneous functions. All meeting this function relationship are resolved by Equation 6,7 and 8 according to the format of homogeneous functions relative to variable $\tau$. This method is utilized not only in elastodynamics (Cherepanov, 1979; Kostrov, 1964; Freund, 1998; Nian-Chun et al., 2004; 2005; 2006; Atkinson, 1965; Hoskins, 1979; Lu et al., 2010a; 2010b; 2010c), but also in elastostatics (Muskhelishvili, 1977; Sih, 1977b; Kanwal and Sharma, 1976; Sneddon, 1951), so much as in the else region (Muskhelishvili, 1977; Galin, 1953).

Introducing the ways of the self-similar functions is capable of gaining analytical solutions of dynamic expansion problems on mode III semi-infinite crack edges subjected to moving increasing loads $\mathrm{Px} / \mathrm{t}$ and $\mathrm{Pt}^{2} / \mathrm{x}$, respectively. This is regarded as the analogous class of dynamic problem of the elasticity theory. The measure of solution is based exclusively on techniques of analytical-function theory and is simple and concise. By making some observations regarding the solution of the mixed boundary-value problem, this has fairly reduced the amount of the calculative work needed to settle such a crack extension problem.

\section{REFERENCES}

Andrew, D.J., 1976. Rupture velocity of plane strain shear cracks. J. Geophys. Res., 8: 5679-5687. DOI: 10.1029/JB081i032p05679 
Atkinson, C., 1965. The propagation of a brittle crack in anisotropic material. Int. J. Eng. Sci., 3: 77-91. DOI: 10.1016/0020-7225(65)90021-2

Atkinson, C., 1975. On the dynamic stress and displacement field associated with a crack propagating across the interface between two media. Int. J. Eng. Sci., 13: 491-506. DOI: 10.1016/00207225(74)90066-4

Broberg, K.B., 1960. The propagation of a brittle crack. Arch. Fur Fysik, 18: 159-192.

Cherepanov, G.P., 1979. Mechanics of Brittle Fracture. 1st Edn., McGraw Hill International Book Company, New York, ISBN-10: 0070107394, pp: 939.

EGMH, 2002. Mathematics Handbook. 1st Edn., Advanced Education Press, Beijing, pp: 300.

Erdogan, F., 1968. Crack Propagation Theories. 1st Edn., Academic Press, New York, pp: 94.

Eringer, A.C., 1975. Elastodynamics. Volume 2. Linear Theory. 1st Edn., YoYo Media.

Fedelinski, P., M.H. Aliabadi and D.P. Rooke, 1997. The time-domain DBEM for rapidly growing cracks. Int. J. Num. Meth. Eng., 40: 1555-1572. DOI: 10.1002/(SICI)10970207(19970515)40:9<1555::AIDNME126>3.0.CO;2-B

Freund, L.B., 1998. Dynamic Fracture Mechanics. 1st Edn., Cambridge University Press, Cambridge, UK., ISBN-10: 0521629225, pp: 563.

Gahov, F.D., 1966. Boundary-Value Problems. 1st Edn., Courier Dover Publications, ISBN-10: 0486662756, pp: 561.

Galin, L.A., 1953. Contact Problems in Elasticity Theory. 1st Edn., GITTL, Moscow.

Gao, H.J., 1996. A theory of local limiting speed in dynamic fracture. J. Mech. Phys. Solids., 44: 14531474. DOI: 10.1016/0022-5096(96)00038-5

Hoskins, R.F., 1979. Generalised Functions. 1st Edn., E. Horwood, ISBN-10: 0853121052, pp: 192.

Kalthoff, J.F., J. Beinert and S. Winkler, 1977. Measurements of Dynamic Stress Intensity Factors for Fast Running and Arresting Cracks in Double-Cantilever-Beam Specimens. In: Fast Fracture and Crack Arrest: A Symposium, Hahn, G.T. and M.F. Kanninen (Eds.), ASTM International, pp: 161-176.

Kanwal, R.P. and D.L. Sharma, 1976. Singularity methods for elastostatics. J. Elasticity, 6: 405-418. DOI: 10.1007/BF00040900

Knauss, W.G., 1987. Dynamic fracture. Int. J. Frac., 25: 35-91.
Kobayashi, A.S., 1979. Dynamic Fracture Analysis by Dynamic Finite Element Method: Generation and Prediction Analyses. In: Nonlinear and Dynamic Fracture Mechanics. Perrone, N. (Ed.), ASME, New York, pp: 19-36.

Kostrov, B.V., 1964. Self similar problems of propagation of shear cracks. J. App. Math. Mech., 28: 1077-1087.

Lu, N.C., Y.H. Cheng, X.G. Li and J. Cheng, 2010 b. Dynamic propagation problem of mode I semiinfinite crack subjected to superimpose loads. Fati. Frac. Eng. Mater. Strut., 33: 141-148. DOI: 10.1111/j.1460-2695.2009.01418.x

Lu, N.C., Y.H. Cheng, X.G. Li and J. Cheng, 2010c. Asymmetrical dynamic propagation problems concerning mode III interface crack. Comp. Interf., 17: 37-48. DOI: 10.1163/092764409X12580201111548

Lu, N.C., Y.H. Cheng, Y.T. Wang and J. Cheng, 2010a. Dynamic fracture of orthotropic solids under antiplane shear loading. Mech. Adv. Mater. Struct., 33: 141-148. DOI: 10.1080/15376490903556618

Muskhelishvili, N.I., 1953. Singular Integral Equations. 2nd Edn., P. Noordhoff, pp: 447.

Muskhelishvili, N.I., 1977. Some Basic Problems of the Mathematical Theory of Elasticity. 4th Edn., Springer, ISBN-10: 9001607012 , pp: 768.

Nian-Chun, L., C. Jin and C. Yun-Hong, 2005. A dynamic propagation problem on the edges of mode III crack subjected to superimpose load. J. Harbin. Inst. Tech., 37: 1093-1097.

Nian-Chun, L., C. Yun-hong and C. Jin, 2004. Studies on some anti-plane problems of a dynamic propagation crack. J. Applied Mech., 21: 156-160.

Nian-Chun, L., C. Yun-Hong, L. Xin-Gang and C. Jin, 2008. Dynamic propagation problems concerning the surfaces of asymmetrical mode III crack subjected to moving loads. Applied Math. Mech., 29: 1279-1290. DOI: 10.1007/s10483-008-1003-z

Nian-Chun, L., C. Yun-hong, T. Xiu-bo and C. Jin, 2006. Analytical solutions to Mode III crack propagation under the action of variable loadings. J. Harbin. Inst. Tech., 38: 1310-1313.

Ranjith, K. and R. Narasimhan, 1996. Asymptotic and finite element analyses of mode III dynamic crack growth at a ductile-brittle interface. Int. J. Fracture, 76: 61-77. DOI: 10.1007/BF00034030

Ravi-Chandar, K. and W.G. Knauss, 1984b. An experimental investigation into dynamic fracture: II. Microstructural aspects. Int. J. Frac., 26: 65-80. DOI: 10.1007/BF01152313 
Ravi-Chandar, K. and W.G. Knauss, 1984a. An experimental investigation into dynamic fracture: I. Crack initiation and arrest. Int. J. Frac., 25: 247-262. DOI: $10.1007 / \mathrm{BF} 00963460$

Ravi-Chandar, K. and W.G. Knauss, 1984c. An experimental investigation into dynamic fracture: III. On steady-state crack propagation and crack branching. Int. J. Frac., 26: 141-154. DOI: 10.1007/BF01157550

Ravi-Chandar, K. and W.G. Knauss, 1984d. An experimental investigation into dynamic fracture: IV. On the interaction of stress waves with propagating cracks. Int. J. Frac., 26: 189-200. DOI: 0.1007/BF01140627

Rosakis, A.J., O. Samudrala and D. Coker, 1999. Cracks faster than the shear wave speed. Science, 284: 1337-1340. DOI: 10.1126/science.284.5418.1337

Rubin-Gonzalea, C. and J.J. Mason, 2000. Dynamic stress intensity factors at the tip of a uniformly loaded semi-infinite crack in an orthotropic material. J. Mech. Phys. Sol., 48: 899-925. DOI: 10.1016/S0022-5096(99)00063-0

Sih, G.C., 1968. Some elastodynamic problems of cracks. Int. J. Frac. Mech., 1: 51-68. DOI: 10.1007/BF00189147

Sih, G.C., 1973. Handbook of Stress-Intensity Factors. 1 st Edn., Lehigh University, Institute of Fracture and Solid Mechanics, pp: 22.

Sih, G.C., 1977a. Mechanics of Fracture 1: Methods of analysis and solutions of crack problems. Noordhoff, Leyden.

Sih, G.C., 1977b. Elastodynamic Crack Problems. 1st Edn., ISBN-10: 9028601562, pp: 404.
Sih, G.C., 1991. Mechanics of Fracture Initiation and Propagation: Surface and Volume Energy Density Applied as Failure Criterion. 1st Edn., Kluwer Academic, ISBN-10: 0792308778, pp: 410.

Sih, G.C., B. MacDonald, 1974. Fracture mechanics applied to engineering problems-strain energy density fracture criterion. Eng. Frac. Mech., 6: 361386. DOI: 10.1016/0013-7944(74)90033-2

Sneddon, N.I., 1951. Fourier Transform. 1st Edn., McGraw-Hill, New York, pp: 542.

Srolovitz, D.J. and L.R. Source, 1997. Dynamic simulation of dislocation microstructures in mode III cracking. Acta Materialia, 45: 3745-376. DOI: 10.1016/S1359-6454(97)00029-3

Tang, X.S., G.C. Sih, 2004. Kinetics of microcrack blunting ahead of macrocrack approaching shear wave speed. Theoretical Applied Fracture Mechan., 42: 99-130. DOI: 10.1016/j.tafmec.2004.08.001

TOMTU, 1994. Advanced Mathematics (Vol.1). 1st Edn., Advanced Education Press, Beijing.

Wang, Y.H., 1992. Analysis of an edge-cracked body subjected to a longitudinal shear force. Eng. Frac. Mech., 42: 45-50. DOI: 10.1016/00137944(92)90275-J

Wang, Y.H., Y.K. Cheung and C.W. Woo, 1992. Antiplane shear problem for an edge crack in a finite orthotropic plate. Eng. Frac. Mech., 42: 971-976. DOI: 10.1016/0013-7944(92)90136-3

$\mathrm{Wu}$, K.C., 2000. Dynamic crack growth in anisotropic material. Int. J. Frac., 106: 1-12. DOI: 10.1023/A:1007621500585 